to a less extent than when inflammation could be kept up by menstrual congestion. In fact, the recurrence of inflammation of the uterine mucous membrane after the menopause is to be explained by cessation of menstruation not having been followed by the involution of the sexual organs.

I have hitherto spoken of the involution of the sexual organs as an uniform process, but it may be only partially effected. Thus, if after the menopause there remains a remnant of internal metritis while the cervix is healthy, the latter may so shrink as to render the cervical canal impermeable to the fluids secreted by the diseased lining of the body of the womb, so as to cause a considerable amount of uterine tormina. This is the explanation of the cases long ago described by Dr. Mathews Duncan as "leucorrhoea of old women". I shall not consider these cases, because I have not met with more than five during the last twenty years; and I have never once had occasion to puncture the cervical canal to afford an exit to the pent-up fluid-an operation that has been not unfrequently resorted to by Dr. Mathews Duncan.

Having thus cleared the ground, I shall briefly draw your attention to the only uterine inflammatory disease that I have found to be of frequent occurrence after the menopause; that is, a more or less acute inflammation of the mucous membrane lining the cervix, and the vagina; for, although cervicitis and vaginitis may be separately met with, cervicitis generally brings on vaginitis-vaginitis being much more severe near the os uteri; and sometimes an ulcer surrounding the os uteri extends to the neighbouring folds of the vagina. This ulceration is not a very serious matter in itself, and may exist for years without coming to worse; but it gives important evidence of the cervical lining being in a similar morbid state, and shows the urgency of applying some kind of caustic to this diseased membrane.

When these affections occur after the menopause, they are less acute, but much more difficult to cure, than before the change. The same amount of cervical ulceration after the change does not wake up the distressing symptoms with which we often have to contend at a previous period of life. This may be partly due to the lowered tone of vital activity, partly to the fact that the submucous tissues of the cervix do not participate in the inflammation. Nevertheless, inflammation of the cervical and vaginal mucous membranes is often attended by a considerable amount of pelvic heat and pain, by back-pain and inability to walk, ty a muco-purulent discharge, disturbance of the vesical functions, and an aggravation of any nervous phenomena to which the patient had been previously liable.

With regard to the rate of activity of vital phenomena after the menopause, it may be safely said that, while heteromorphous tissues often grow rapidly, inflammation is singularly chronic. The sluggishness of the tendency to repair on the part of the inflamed mucous mem. brane may be to a certain extent measured by the comparative length of time that an eschar will take to fall from the mucous membrane, according as the caustic is applied before or after the menopause. Thus when, after the change, the solid nitrate of silver is applied to the ulcerated os, the eschar may take nine or ten days to fall off; whereas it is known to fall off, from the fourth to the fifth day when the caustic is applied, under similar circumstances, to younger women. It is, therefore, obvious that a speedy cure should not be promised.

I have fully explained how defective involution of the sexual organs renders them liable to inflammation; and when, at the menopause, I find that a patient has a hard and an enlarged cervix, instead of encouraging her to believe that she is to suffer no more, I take care to tell her that she is likely to have occasional relapses for many years to come, if she be not careful. These relapses have been attributed to a chill, to overwalking, to too frequent coition (for in some women there seems to be no limit of age for the continuance of matrimonial habits). I have noticed these attacks to recur in spring and autumn in some patients.

Exceptional cases of inflammation of the sexual mucous membrane after the menopause can only be explained by a cachectic tendency to disease, on the part of all the mucous membranes. In these women, there is an habitually congested state of the vagina and of the cervical mucous membrane, with an atonic ulcer round the os uteri, which does not always admit of being permanently healed. In one case of this description, the prolonged application of a solution of nitrate of silver every fifth day did not cure the patient; and I only now have recourse to surgical treatment when the symptoms become worse and the discharge purulent.

The following case well exemplifies the difficulty of curing some of these patients. A lady had enjoyed good health, but had a severe attack of uterine inflammation, after being confined of her last child at thirty-five. She occasionally suffered from it, until menstruation ceased at forty-six ; and, when forty-eight, she had a severe attack, from walking on wet grass. Several applications of nitrate of silver did no good; and, when she passed through town, I repeatedly ascertained that there was always an angry patch of ulceration, of about the size of a shilling, on the posterior lip of the os uteri. As this interfered very little with the patient's comfort, she did nothing until there came a very severe and almost constant pain in the back and right lumbar nerves. I then made two successive applications of the acid nitrate of inercury ; but the ulcer was just the same when she came to me three months afterwards. I then applied the potassa fusa cum calce; but the patient started for Ben Rhydding before the ulcer was healed. There she underwent a long course of hot and cold water treatment, which had no effect on the spinal neuralgia; and when she passed through town to take the Bath waters, I still found the ulcer, though smaller and less angry.

This case gives an idea of the difficulties sometimes met with, although they were doubtless increased by the patient's objection to being controlled.

The practical hints to be derived from this paper are : I. That the occasional recurrence after the menopause of inflammation of the sexual mucous membranes may be safely predicted to those who have long been martyrs to inflammation of the entire womb, when they presume too much on their partly recovered strength ; for I have always found this to occur whenever I have had the opportunity of watching such patients for the ten years that followed cessation: 2. That we may safely predict inflammation of the sexual mucous membranes to those who overtax their strength after the menopause, whenever the uterine tissues are being constantly teased by a small interstitial fibroid or a polypus, when the cervix is hypertrophied or of a fibroid consistency, and also when women are cachectic and have always one or more of their mucous membranes in a diseased condition.

\section{CASE OF JAUNDICE.}

\section{By WILliaM DATE, Crewkerne.}

J. C., aged 35 , a stalwart, muscular, tolerably temperate agricultural labourer, has always enjoyed good health up to the present attack. In the evening of March I8th, 1872, he complained of headache. The following morning the headache was more severe : he said he could not see well, and remained in bed. Next day he felt better and got up, but did not go to his work. In the evening I saw him.

March 2oth (Evening). He was throwing himself about the bed, moving incessantly, and talking wildly. When spoken to authoritatively, he became quiet for a minute and answered questions, but if left alone he speedily relapsed into delirium. He complained of intense pain in the head, horrible dreams, and dimness of sight. He said he could see objects, but they seemed wrapped in a cloud. He shrunk. from a bright light. His pupils were contracted; the face pallid; lips leaden coloured, giving the countenance a cadaverous appearance. The tongue was thickly coated with white fur. Pulse 120 ; respirations 30 ; temperature 104.6 deg. Bowels freely moved in consequence of medicine previously administered. The urine could not be observed.

March 2Ist (Morning). The pain in the head was better, but not gone ; he was very drowsy, but more rational. He had some restlesz unquiet sleep. Free purging continued; the motions were dark and offensive. The urine was copious and natural in colour. Pulse I02: respirations 28 ; temperature $103 \mathrm{deg}$.

March 22nd. He had had some quiet sleep. IIe said his head felt heavy and "queer"; the pain was quite gone. He could see well again; the pupils were more active. He had passed two motionsthe last one very light in colour. Urine rather yellow; skin slightly tinged with yellow. There was no tenderness nor enlargement of the liver. He vomited once this morning for the first time. Pulse IOo; respirations 24 ; temperature 102.6 deg.

March 25 th. There was no return of headache. He complained of tenderness in the calves of his legs. The skin and urine were of $a$ deeper yellow colour. Careful examination revealed nothing abnormal about the liver. The motions were costive and white; the tongue brown and dry. Pulse IIO; respirations 28 ; temperature 102.7 deg.

March 28th. The skin had a deep yellow colour; the urine was very dark. There was no tenderness nor alteration in the size of the liver. The tongue was cleaner. Pulse 88 ; respirations 21 ; temperature 99.8 degrees.

It is useless to occupy space with further notes. From this time the case progressed as one of simple functional jaundice, and made an uninterrupted but rather protracted recovery. The interest centres entirely in the first three days of attendance. During that time there was not one single symptom indicative of jaundice or of disease of the liver. The intense pain in the head, delirium, disturbed vision, intolerance 
of light, and high temperature, pointed rather to some meningeal inflammation. It was not until the third day of attendance that any sign of jaundice appeared ; and pari passu with the development of jaundice the other symptoms became ameliorated. The man was moderately temperate ; there was no history of any recent debauch, nor of a blow on the head, nor of any emotional excitement.

Now it is an interesting question to determine the mutual relation in this case of the head symptoms on the one hand, and the jaundice on the other. It is said that violent emotion will produce jaundice. If so, it must be possible that the brain affection may have been the primary cause, and the jaundice have resulted from an abnormal exercise of nerve-force, at present ill-understood. On the other hand, signs of cerebral disturbance are sometimes very prominent in cases of jaundice. But, then, how are we to account for the urgent head-symptoms and the high temperature appearing so long before any external evidence of jaundice? Altogether, the question of cause and effect is a puzzling one, which I leave to your readers to answer.

Note. Sept. 7th. -I have seen J. C. to-day. There is now no trace of jaundice; he has regained his lost flesh and strength, and feels quite well, except that if he work in the sun he becomes very giddy.

\section{CLINICAL MEMORANDA.}

\section{T O N G U E - S U C K I N G.}

Dr. Morris's communication on Spontaneous Dislocation of the Jaw through Tongue-sucking is very interesting, and will become more so if Dr. Morris will kindly put upon record also what is the physical and mental condition of the young lady to whom the accident occurred.

I have taken some pains during the last twelve years to invite the attention of the profession to the blighting effects upon the infant of fruitless sucking, and to show that the retained habits of sucking-viz., tongue-sucking, thumb-sucking, etc. -and the deformities of the jaws resulting therefrom, are evidences of the individual having been ex. posed during infancy to this baneful error of management. I think it is likely that facts recorded by some other pen than my own may assist in directing attention to this important subject.

September 2 nd, 1872 . Thomas Ballard, M.D.

\section{N G R O IV I N G N A I L.}

I DESIRE to add a mite to the evidence repeatedly given in the JOURNAL that the removal of the nail (to my knowledge not always successful) is unnecessary.

About twenty years ago, I applied a bit of compressed sponge to afford tempurary relief, and was delighted to find that it effected a radical cure. I make the sponge as solid as leather, by wetting and then winding string very tightly round it, and drying it thoroughly. Of this I cut a small pyramidal piece, less than a grain of rice. This I insert beneath the nail, and secure it by strips of adhesive plaster, applied longitudinally, to avoid compression. The sponge soon becomes moist and swollen, keeping the nail from the irritated flesh. Any granulations should previously be destroyed with strong nitric acid. I have adopted this plan upon many occasions, and have never found it to fail.

Liverpool, September 4 th, 1872 . BenjaMin Blower.

\section{OBSTETRIC MEMORANDA.}

\section{ERGOT IN ABORTION.}

IN a discussion at the late meeting of the British Medical Association, Dr. Evory Kennedy remarked that, " in abortion, ergot was generally inadvisable." As abortion about the tenth or twelfth week is frequently met with in general practice, it is, I think, important that the question of the utility or uselessness of ergot in cases of this kind should be freely ventilated. I have given the drug in above two hundred cases of profuse haemorrhage with threatened abortion, and with the happiest result. Only yesterday, I attended a woman at the full time with a healthy boy. On February Ioth, twenty-five weeks ago, she was taken with great flooding and alarming faintness. She was in the third month of pregnancy, having been "unwell" last in November. I plugged at once with a handkerchief, not being quite able to bring away the ovum, which was protruding to a great extent at the cervix. I then gave doses of the ammoniated tincture of ergot every hour. All bleeding ceased, and the patient went on perfectly well to her full time. I am engaged to attend a lady in October who had precisely the same symptoms in the spring. Indeed, I can call to mind several instances where, ergot being given to check uterine hæmorrhage, it has acted well by causing, not the expulsion of the ovum, but its gradual retrocession into the uterus. From what $I$ have seen of the use of the remedy, I am prepared to maintain that it is a most valuable help ; but that not unfrequently it does good, not by forcing the contracting uterus to expel its contenis, but often, contrary to our expectations, by helping the organ to retain its precious charge to its ultimate preservation. Ergot, in my opinion, is, in threatened abortion, only second to a plug in the vagina; and the best plug I find is a piece of sponge. Stourbridge, August 26th, I872. AlFred FreER, M.R.C.S.

\section{REPORTS}

\section{MEDICAL AND SURGICAL PRACTICE IN THE HOSPITALS OF GREAT BRITAIN.}

\author{
LONDON HOSPITAL.
}

Cases under the Care of Mr. Hutchinson.

Buffer-crusti: Rupture of Liver and Splcen : Extravasation of Blood into the Peritoneum: Almost entire Absence of Sympioms for several Hours after Accident: No alarming Symptoms for the first Forty-eight Hours: Death in about Seventy-six Hours.- James Wall, aged I9,
was struck in the abdomen above the umbilicus by the buffers of a railway engine, in the afternoon of May 16 th. He was not faint immediately after the accident, and was able to walk to a doctor's house without assistance. Two hours after the accident, he was somewhat worse. When admitted in the evening of the same day, he was cold and suffering somewhat from shock, but not to any degree that appeared serious. He shortly afterwards vomited, bringing up nothing except the proper contents of the stomach. The same evening he passed urine quite free from blood. Next day (i 7 th), he vomited two or three times, but always expressed himself as "better"; there were no symptoms which appeared serious. On the I $8 \mathrm{th}$, he was still sick occasionally, but said that he felt better than the day before. He had, however, some pain in the abdomen, and considerable tympanitis. He was constipated, and had been so since admission, in spite of purgative medicine and an enema; perhaps this symptom was caused by the opium (twenty-five minims of tincture) which was administered on the evening of admission and on the following day. On the igth, he was worse ; the face dusky and anxious; the abdomen was more tympanitic and more tender on pressure; breathing entirely thoracic; vomiting continued. He sank gradually, and died at IO P.M., about seventy-six hours after the accident.

At the post mortem examination, a rupture was found on the under surface of the right lobe of the liver. Much bile was extravasated on the surrounding parts of the surface of the liver. Its upper surface was much ecchymosed. There was also a laceration of the spleen, extending from its edge for an inch into its substance, and coagulated blood was attached somewhat firmly to this wound. The peritoneum contained a large quantity of blood, which was fluid. There was a little recent lymph on the surface of the liver, and slight congestion along the edges of the intestinal coils, but no other signs of peritonitis. All the other abdominal and thoracic organs were healthy.

Black Caries of Tibia, ultimately destruction of almost the entire thickness of the Bone: Fracture and imperfect Union: No Separation of Pieces of Bone: No Formation of New Bone: Ripeatid Operations: Prolonged Rest: Yodide of Potassium: Bencfit, but no Cure: Ampiutation through Leg.-Charles R., aged 32, a gunsmith, was probably the sub. ject of hereditary syphilis; his upper central incisors are screw.driver shaped, but not quite conclusive as to syphilitic taint. His skin is pale and earthy-looking. There is neither history nor evidence of acquired syphilis. His left tibia had been troubling him for about three years and a half, at which date the lower part of the leg inflamed, a swelling formed over the bone, and, after it had broken, numerous bits of bone (about, he says, as large as finger-nails), came away. He believed that this inflammation and disease of bone were caused by his having fallen and twisted the leg a year before; for ever after the fall his leg gave him some trouble, though not enough to prevent him from working. About a year after the formation of the abscess and escape of dead bone, he was under Mr. Curling's care, and had some dead bone removed. After this, the ulcer nearly healed, and he got on well for another twelve months. It then again began to trouble him, and another piece of bone (he said, as large as a finger) came away. The limb became more and more troublesome, till admission on April I $8: \mathrm{h}$, 